Cytokines and SIDS. Pro-inflammatory cytokines are found in the brainstem of infants dying from SIDS, and an increase in IL-6 has been demonstrated in the CSF. The limitations of these findings in the etiology of SIDS is discussed by Waters KA. (Lancet Neurology February 2004;3:81).

\title{
NEONATAL CEREBRAL INFARCTION AND NEUROMOTOR DYSFUNCTION
}

Twenty-two children with cerebral infarction on neonatal MRI were examined neurologically at school age in a study at Imperial College School of Medicine, Hammersmith, London, UK. Six (30\%) had hemiplegia and an additional $7(30 \%)$ had neuromotor abnormalities, including asymmetries. The remaining 9 had normal motor function. Hemiplegia occurred only in patients showing neonatal MRI evidence of hemisphere, internal capsule, and basal ganglia involvement. (Mercuri E, Barnett A, Rutherford $\mathrm{M}$ et al. Neonatal cerebral infarction and neuromotor outcome at school age. Pediatrics January 2004;113:95-100). (Respond: Eugenio Mercuri MD, Department of Paediatrics, Imperial College School of Medicine, Hammersmith, London, UK).

COMMENT. In children suffering a neonatal cerebral infarction, signs of neuromotor impairment become more obvious at school age when the neurologic examination is more structured.

Risk of recurrent stroke in children and antiphospholipid screening are reviewed from the University of California, San Francisco (Fullerton HJ, von Scheven E. Editorial. Neurology January (2 of 2);62:172-173; and Lanthier S et al. Neurology 204;62:194-200). Lanthier et al found no difference in recurrence rates between the anticardiolipin antibody (aCL)-positive and aCL-negative groups, when 185 children with a first arterial ischemic stroke or TIA were followed for a median of 3 years. However, aCL-positive children were more likely to be treated with antithrombotic agent, and treated patients were less likely to have a recurrence. In the absence of a prospective randomized trial, screening for antiphospholipid syndrome (APS) should continue as a routine component of the evaluation of arterial ischemic stroke and risk of recurrence.

Subsequent publication of the results of the antiphospholipid antibodies and stroke study (APASS), a prospective double-blind cohort study involving 1770 participants from multiple US clinical sites (JAMA Feb 4, 2004;291:576-584), concludes that the presence of $\mathrm{aCL}$ in patients with ischemic stroke does not predict an increased risk of subsequent stroke. Routine screening for aPL in patients with ischemic stroke may not be warranted. The debate continues.

\section{SEIZURE DISORDERS}

\section{EFFECTS OF AEDS ON SERUM NITRITE AND NITRATE LEVELS}

Serum nitrite and nitrate levels were determined in 34 epileptic children treated with valproic acid and 23 with carbamazepine and compared to 38 non-active epileptic children 
with no antiepileptic drug therapy, in a study at KSU Medical School, Turkey. Nitrite and nitrate levels, metabolites of nitric oxide, were significantly higher in both valproic acid and carbamazepine-treated groups compared to controls $(\mathrm{p}<0.01)$, and nitrate but not nitrite was significantly higher in the valproic acid-treated group compared to the carbamazepine group $(\mathrm{p}<0.01)$. Serum drug levels did not correlate with nitrite and nitrate levels. The authors suggest that valproic acid and carbamazepine might exert an antiepileptic effect through nitric oxide. (Karabiber H, Yakinci C, Durmaz Y et al. Serum nitrite and nitrate levels in epileptic children using valproic acid or carbamazepine. Brain Dev January 2004;26:15-18). (Respond: Dr Hamza Karabiber, KSU Medical School, Department of Pediatrics, 46050 Kahramanmaras, Turkey).

COMMENT. Nitrite and nitrate levels are used as indicators of nitric oxide (NO) levels in serum. NO has been found to have an anticonvulsant effect in animal studies. The above report suggests that the anticonvulsants, valproic acid and carbamazepine, may have antiepileptic effects through $\mathrm{NO}$ accumulation.

Valproic acid (VPA) blood gene expression patterns and epilepsy. The expression of 461 genes was altered in VPA-treated compared with drug-free children with epilepsy, in a study at the University of Cincinnati (Tang Y et al. Acta Neurologica Scandinavica March 2004;109:159-168). A new mechanism of action of VPA is suggested that involves downregulation of serine threonine kinases and a separate genomic profile that correlates with VPA-induced seizure freedom.

\section{MEMORY PERFORMANCE WITH INTRACTABLE EPILEPSY}

The cognitive and psychological predictors of everyday memory were investigated in 37 children (mean age 13.7 years, range 7.3-17.9 years) with medically intractable epilepsy treated at the Hospital for Sick Children, Toronto, Canada. Only a parent-report measure of attention (Child Behavior Checklist Attention) significantly predicted everyday memory ratings. Standard tests of attention, intelligence, visual and verbal memory, working memory, and mood/emotional state were not predictive of everyday memory. (Kadis DS, Stollstorff M, Elliott I et al. Cognitive and psychological predictors of everyday memory in children with intractable epilepsy. Epilepsy Behav 2004;5:37-43). (Respond: Dr Darren S Kadis, Hospital for Sick Children, Toronto, Ontario, Canada).

COMMENT. Everyday memory deficits in children with epilepsy are secondary to attentional problems. They differ from that of adults whose everyday memory correlates with standard psychological measures. Memory deficits in adults with epilepsy have also been correlated with anxiety and/or depression, age and duration of the seizure disorder.

Memory impairment with febrile seizures. Adult rats that had been subjected to repetitive brief, experimental febrile seizures (FS) at days 10 to 12 postpartum, showed long-term memory deficits as assessed by the Morris water maze test (Chang Y-C et al. Ann Neurol Dec 2003;54:706-718). These results raise concerns about long-term cognitive consequences of even brief repetitive FS during infancy. Despite limitations, immature animal experimental FS can provide useful correlations with the clinical FS, its mechanism and 